Journal of Zhejiang University-SCIENCE B (Biomedicine \& Biotechnology)

ISSN 1673-1581 (Print); ISSN 1862-1783 (Online)

www.jzus.zju.edu.cn; www.springerlink.com

E-mail: jzus@zju.edu.cn

\title{
Editorial:
}

\section{Lymphatic vessels, miRNAs, and CAR T cells in tumor immunology}

\author{
Xinguo JIANG \\ VA Palo Alto Health Care System, Stanford University School of \\ Medicine, Palo Alto, CA 94304, USA \\ E-mail: xinguoj@stanford.edu
}

https://doi.org/10.1631/jzus.B1910003

This special feature contains three review articles that summarize recent advances pertaining to tumor immunobiology. Normalization of antitumor immunity through checkpoint inhibitors has achieved significant clinical success and benefited many cancer patients. However, not all cancer patients respond to these treatments, and among the responders, some may develop resistance and others may suffer autoimmunity that requires intervention. Tumor immunotherapy holds promise for further improving the survival of cancer patients, but deeper understanding of immunological networks that regulate anti- and pro-tumor immunity is needed. The review papers collected in this issue cover a few topics that may stimulate future interest in the relevant research field.

It was well-recognized that the lymphatic vasculature provides a viable conduit for tumor metastasis, and metastasis toward drainage lymph nodes is often considered a sign of tumor invasiveness. Jiang (2020) discusses the most recent advances in how the lymphatic vasculature and lymph nodes may have new roles in mediating anti-tumor immunity in conjunction with immunotherapy, a notion that has been traditionally neglected.

Tumor-associated macrophages (TAMs) are important innate immune cells that shape the tumor microenvironment. On most occasions, these promote immune tolerance, and therefore may induce resistance to checkpoint therapy. Chen et al. (2020) discuss the

(b) ORCID: Xinguo JIANG, https://orcid.org/0000-0003-3266-9238 (c) Zhejiang University and Springer-Verlag GmbH Germany, part of Springer Nature 2020 role of microRNA (miRNA) in modulating macrophage biology in tumors. They first summarize miRNAs that are involved in macrophage differentiation and polarization, and then describe how miRNAs packaged in exosomes can transfer biological instructions between macrophages and tumor cells. This tends to foster macrophage M2 polarization and escalate tumor cell invasiveness and metastasis. The article concludes by examining the clinical potential of macrophage-related miRNAs as diagnostic tools as well as therapeutic targets.

Whereas checkpoint inhibitors promote antitumor immunity through lifting the brake that blocks the functional immune response, chimera antigen receptor $\mathrm{T}(\mathrm{CAR} T)$ cells are engineered to directly promote $\mathrm{T}$ cell function for killing tumor cells. CAR T-cell therapy has recently entered clinical stages to treat acute lymphocytic leukemia and diffuse large B-cell lymphoma, indicating that CAR T-cell therapy may represent a promising way to treat hematological malignancies. Huang et al. (2020) describe current advances in using CAR T strategy to treat refractory/ relapsed multiple myeloma, a B-cell malignancy. The authors summarize candidate antigens that have the potential to be used for CAR T engineering; the article also discusses how potential off-target toxicities derived from CAR $\mathrm{T}$ therapy can be avoided or treated.

The principles highlighted in this collection of review articles will have applications to preclinical research as well as clinical practice. As such, these papers will enrich the literature of this exciting and fast evolving field of immuno-oncology.

\section{References}

Chen C, Liu JM, Luo YP, 2020. MicroRNAs in tumor immunity: functional regulation in tumor-associated macrophages. $J$ Zhejiang Univ-Sci B (Biomed \& Biotechnol), 21(1):12-28. https://doi.org/10.1631/jzus.B1900452

Huang H, Wu HW, Hu YX, 2020. Current advances in chimeric 
antigen receptor T-cell therapy for refractory/relapsed multiple myeloma. J Zhejiang Univ-Sci B (Biomed \& Biotechnol), 21(1):29-41.

https://doi.org/10.1631/jzus.B1900351
Jiang X, 2020. Lymphatic vasculature in tumor metastasis and immunobiology. J Zhejiang Univ-Sci B (Biomed \& Biotechnol), 21(1):3-11.

https://doi.org/10.1631/jzus.B1800633 